\title{
A novel energy-aware cluster head selection based on particle swarm optimization for wireless sensor networks
}

\author{
Buddha Singh ${ }^{*}$ and Daya Krishan Lobiyal
}

\author{
* Correspondence: b.singh.jnu@ \\ gmail.com \\ School of Computer \& Systems \\ Sciences, Jawaharlal Nehru \\ University, New Delhi, India
}

\begin{abstract}
The wireless sensor networks have long been an attractive field to the researchers and scientists for its ease in deployment and maintenance. In this research, we focus on the maximization of network lifetime which has become a critical issue in sensor networks. Clustered organization of nodes with aggregation of data at the cluster head becomes one of the significant means to extend life expectancy of the network. This paper proposes Particle Swarm Optimization (PSO) approach for generating energy-aware clusters by optimal selection of cluster heads. The PSO eventually reduces the cost of locating optimal position for the head nodes in a cluster. In addition, we have implemented the PSO-based approach within the cluster rather than base station, which makes it a semi-distributed method. The selection criteria of the objective function are based on the residual energy, intra-cluster distance, node degree and head count of the probable cluster heads. Furthermore, influence of the expected number of packet retransmissions along the estimated path towards the cluster head is also reflected in our proposed energy consumption model. The performance evaluation of our proposed technique is carried out with respect to the well-known cluster-based sensor network protocols, LEACH-C and PSO-C respectively. Finally, the simulation clarifies the effectiveness of our proposed work over its comparatives in terms of network lifetime, average packet transmissions, cluster head selection rounds supported by PSO and average energy consumption.
\end{abstract}

Keywords: Wireless sensor network, Particle swarm optimization, Energy-aware clusters, Intra-cluster distance, Packet retransmission

\section{Introduction}

Wireless sensor networks have revolutionized the recent years of development by creating significant impact throughout the society. The demand for these networks is spurred by various civilian and military applications [1,2]. The sensor network consists of battery-powered devices bestowed with a multitude of sensing modalities. Such networks are usually formed with great bulk of nodes (called sensors) distributed over the sensing area in order to capture maximum coverage [3]. The battery operated sensors are known for their lightweight and economic pricing. The sensors are responsible for sensing certain environmental property and periodically transmitting the related information to the processing station (cluster head or sink). These devices are generally 
deployed in unattended hostile regions, which ultimately makes the power source of the sensors non-renewal (i.e. difficult to recharge). However, in the research there exist a number of relevant energy preserving techniques, which tends to prolong the network lifespan. In this paper, we have considered one such swarm intelligence mechanism known as Particle Swarm Optimization $[4,5]$.

Particle Swarm Optimization (PSO) is an artificial intelligence mechanism which is motivated by the social behaviors of natural species, for instance - herd of animals, swarm of birds, etc. [6]. The population-based swarm intelligence method executes with the aim of optimizing an objective (or fitness) function. The algorithm employs a swarm of search points (also called particles) and tracks the fitness of each particle. Every particle is associated with corresponding velocity. This assists the particle to move onto a better position, if the cost to the objective function is optimized. PSO is known is to perform better than other swarm intelligence techniques (Ant Colony Optimization, Genetic Algorithm, etc.) in terms of computation complexity and convergence rate $[7,8]$. In PSO, the particles possess a fitness criterion for all the positions they visit. Hence, it becomes essential to maintain a local best fitness value for the particles during every generation (iteration). The local information further helps to locate the trajectory towards global best position. The pattern emerged from the collective intelligence of the particles ultimately optimizes the objective function. In this paper, we apply the swarm optimization to find cluster head positions in order to reduce the intra-cluster distance and overall energy consumption during packet transmission to the sink.

The rest of the paper organization is done as follows: Section 2 summarizes the previous work in the related area. The assumptions regarding the network model is provided in section 3. The proposed PSO-based cluster head selection scheme is analyzed in section 4. This is followed by section 5 that presents analytical retransmissions computation along with estimation of average distance between a sensor node and cluster head (intra-cluster distance). In section 6 the proposed energy model is provided to analyze the average power consumption of the network. The simulation and performance modeling is carried out in section 7. Finally we conclude our research work in section 8 .

\section{Related work}

Plenty of research is available in literature to reflect the application of swarm optimization in the context of wireless sensor network. A novel cluster-based approach is introduced in [9] using PSO. The authors have proposed a fitness function to minimize the intra-cluster distance between the sensor nodes and cluster head. The function also helps in optimizing the energy efficiency of the network. The implementation of the PSO is completely centralized and is executed at the base station (sink). Moreover, at the initiation phase of every cluster head selection round, all the sensors in the network are required to send their location information and residual energy level to the sink. Such transmissions in bulk not only increases congestion in the network, but also lead to unnecessary energy drainage. However, the simulation results for the method (PSO-Clustering or PSO-C) have shown outperforming results in comparison with LEACH [10] and LEACH-C [11] protocols respectively. 
An improved PSO has been proposed in [12] for improving the performance of the optimization technique and keep a sense of balance between exploration and exploitation of particles in the swarm. The authors have tried to broaden the exploration ability of particle swarm by using Metropolis Algorithm (MA). In [13] another research work evaluates a routing optimization method on the basis of graph theory and particle swarm optimization algorithm in multi-hop sensor network. The cluster head is elected with a help of a weighted function $\omega(i)$, which is computed iteratively for each ith round. Moreover, routing of packets in the network is optimized with the fitness function that aims at maximizing two topological properties, i.e. transmission distance and residual energy after every round of transmission (to the base station). The work is simulated and compared with the probabilistic method of selecting cluster heads and the experiments showed positive results. Further, the authors in [14] have used PSO to optimize the location of the sensors with an objective to enhance the network connectivity. Two types of optimization schemes are used, namely - single directional and bidirectional approaches. The proposed work also considers the effect of localization errors. However, the simulation results confirm that the proposed scheme executes perfectly for a sparse sensor network. Similar works can also be found in [15-18] that proposed energy efficient layout for better coverage and connectivity in the sensor networks through the application of particle swarm optimization.

In a research conducted in [19] swarm optimization with Dijkstra algorithm is utilized to find the optimal path from initial to target state, in accordance with some welldefined performance criterion. The mobile sensors (or beacon nodes) are assumed to be aware of their dynamically changing coordinates. The simulation of the algorithm showed better performance than some exiting path-finding methods. However, the implementation of the PSO method bears certain impediments in theoretical foundation. In some another recent work in [20] the authors have used swarm optimization method to localize head nodes on the basis of the distance and residual energy of the cluster members. However, the contribution lacks proper estimation of the intra-cluster distance. In yet another work carried out in [21] PSO is applied to optimize the sensor deployment strategy in order to maximize the network coverage in mobile sensor network. The authors also attempt to find the optimal position for cluster heads with the help of a familiar energy model. During the process of coverage optimization, the sensors move to new positions, thereby forming uniformly distributed topology. As compared with Genetic Algorithm (GA), the proposed method worked quite efficiently. However the algorithm is completely executed in a centralized manner, i.e. directly monitored by the base station, which pose as a major drawback in application.

In this paper, we make contributions that are worth in enhancing the lifetime and performance of the sensor network. We proposed a PSO-based cluster head selection scheme to find the fittest position for the head nodes. In a cluster, the head node is known to be located in the best position, if it reduces the intra-cluster distance. Theoretically, the core of the distribution with maximum density is considered to be equidistant from all its boundaries. Therefore, the sole aim of our PSO-based method is to localize the head nodes around the center of cluster density. On the basis of the optimized $\mathrm{CH}$ position we compute the estimated distance covered by packet transmission from a sensor node to its cluster head. Further, we analyze the effect of link failure on transmission of packets and also derived expected number of retransmission over the 
path to cluster head. Finally, energy computation is performed to evaluate the energy savings performed by our proposed technique as compared with the LEACH-C [8] and PSO-C [6] protocols.

\section{Network model \& assumptions}

In this section we describe our network scenario model (Figure 1) used for simulation. The assumptions made regarding the sensor network is provided in the following:

- The wireless sensor network is assumed to be a circular geographic area with the sink $S$, positioned at coordinate $(0,0)$, and radius $R_{s}$.

- The sensor nodes are uniformly deployed in the sensing area $A_{s}$. The number of sensor nodes are distributed according to the two dimensional Poisson point process with $\rho$ as the expected density of nodes in a cluster $A_{C}$.

- The cluster covers a circular region with its cluster head at the center $O$ with radius $R$.

- It is presumed that there are total $k$ clusters in the sensor network. Further, owing to the uniform node deployment strategy, we can compute an approximation for the cluster radius, $R$ :

$$
\begin{aligned}
& k \times A_{C}=A_{S} \Rightarrow k \times \pi R^{2}=\pi R_{S}^{2} \\
& \therefore R=R_{\mathrm{S}} / \sqrt{k}
\end{aligned}
$$

- A sensor is allowed to use different levels of transmission power depending upon its distance from the target node (or the cluster head). The distance can be estimated from the strength of the signal received from the destination node.

- Altering transmission power results in varying transmission ranges $r_{i}(i=1,2,3, \ldots) \cdot r_{\min }$ is considered as the minimum allowable range of transmission for any sensor.

- The base station (or sink) periodically sends a request to the cluster head to upload samples collected by the sensors (Figure 2). On receiving the request, the cluster head broadcasts data-gathering-signal to all its cluster members.

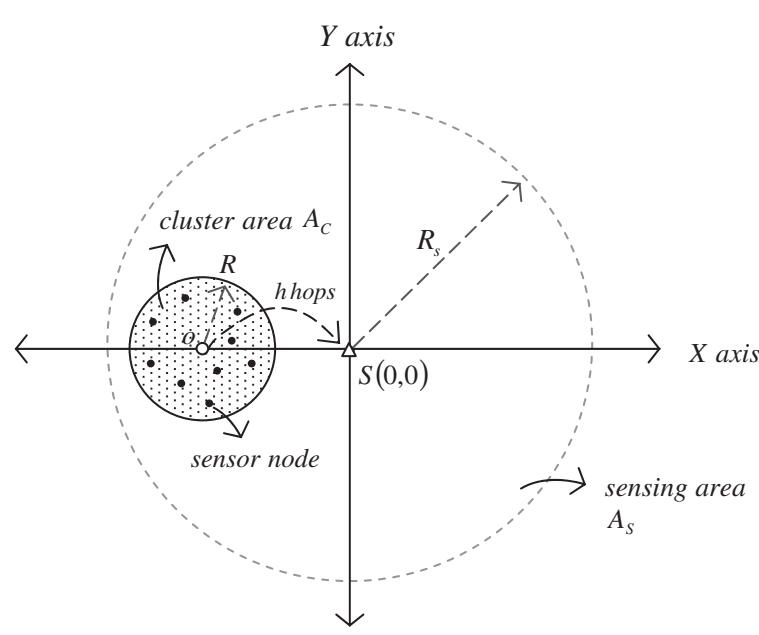

Figure 1 Network Scenario Model. 


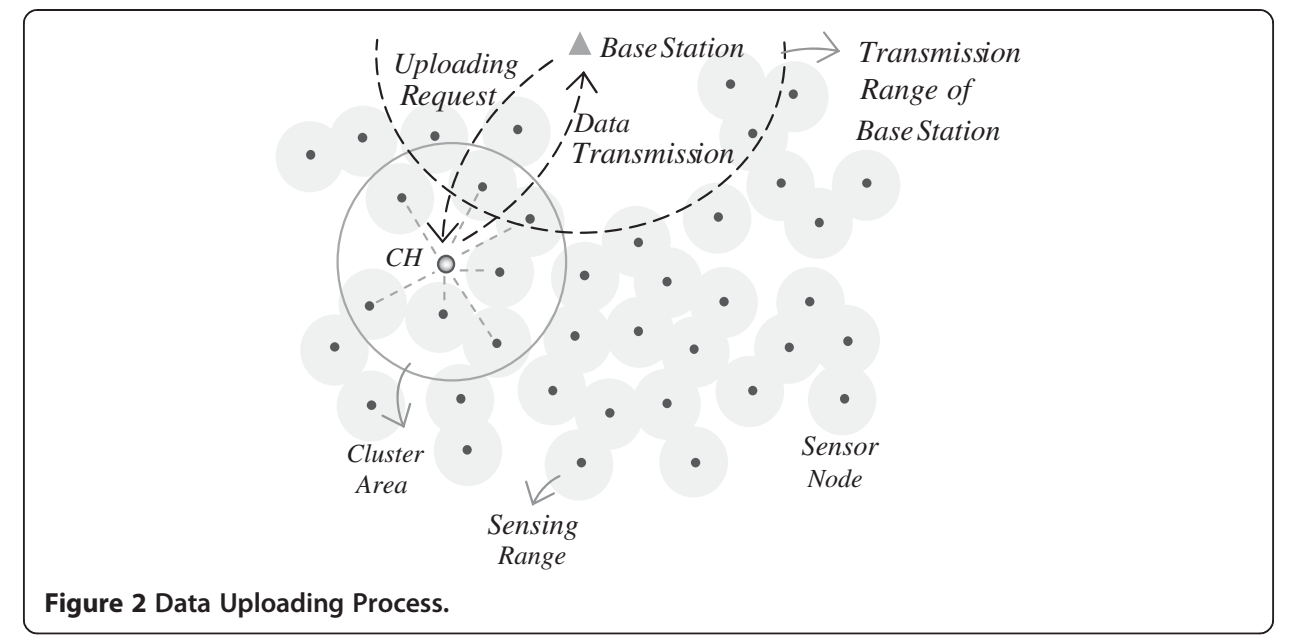

- The member nodes transmit their samples to the cluster head, after which the $\mathrm{CH}$ processes and aggregates the gathered samples and finally forwards the information to the sink.

- The cluster member nodes are able to temporarily switch off its power supply and enter sleep mode to preserve energy waste. Every node uses a probability $\beta_{\mathrm{s}}$ to sleep for certain time span.

- During data transmission, packets are relayed to the cluster head by the intermediate sensors in $h$ hops. Further, it is also assumed that every communication link in the network is associated with a link failure probability $\left(p_{l k}\right)$.

- To compute the energy expended in transmission, we consider maximum number of hops $\left(h_{\max }\right)$ traversed with minimum transmission range $\left(r_{\min }\right)$.

- In our research, we have applied swarm optimization in clustered sensor network, where the nodes are assumed to be stationery. The basic aim is to find optimized position for cluster head, i.e. as near as possible to the center of mass (COM). The mass center (Figure 3) of a cluster can be defined as the mid-position of the sensor distribution within the cluster. Such localization for cluster head would ultimately assist to minimize the average distance covered by the sensors to transmit data to

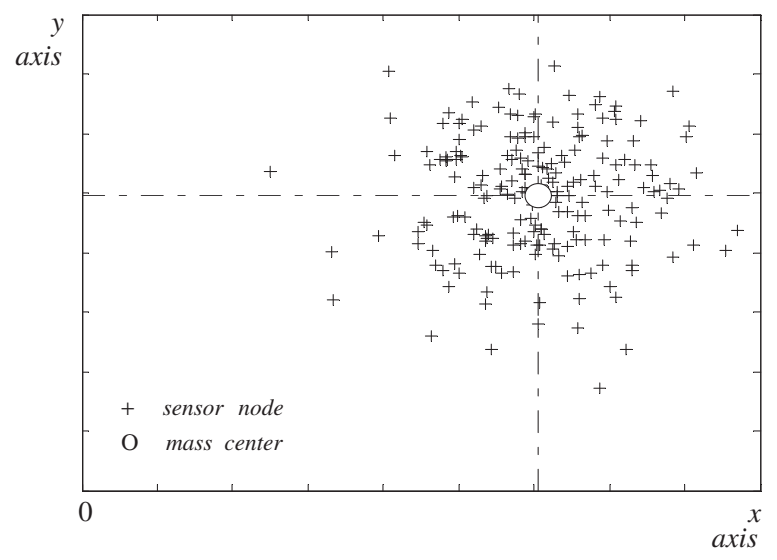

Figure 3 Highlighting Center of Mass (COM) with respect to the Sensor Distribution. 
the cluster head. The swarm intelligence considers $N$ points or particles around the $C O M$ area and iterates in search of the best location.

- Moreover, the velocity of the particle is assumed to be the rate at which the position of the particle is changed, i.e. the sensor does not move but the particle coordinate under consideration is shifted. Also, the sensor node found nearest to any particle is associated with the node's residual energy (particle energy) and head count (particle count). These parameters are required during the evaluation of the objective function for each particle in all iterations.

- In PSO application a centralized coordinator is required to maintain several attributes of the particles for every generation. In order to accomplish the requirement, we randomly select a sensor node in every cluster and appoint it as cluster assistant $\left(n_{C A}\right)$. The cluster assistant is assumed to maintain the local best position of every sensor along with other sensor characteristics, like - current position, particle energy, head count, global best solution and additional PSO parameter values. At the beginning of every round of cluster head selection, all the sensors in a cluster provide the required information to their respective assistant node.

- The cluster assistant node is supposed be the local processing center for PSO computations carried out within a cluster. It does not perform environmental sensing, nor does it participate in data communication to the cluster head. Since, the power expended in computation is quite insignificant than the energy exhausted in communication, $n_{C A}$ survives quite long to function as the processing node. The assistant node prefers to sleep while the selection round is over. The node only awakes when it is informed by the present $\mathrm{CH}$ about its inefficiency to function as the head node.

- However, if the energy falls below certain level the $n_{C A}$ node sends a request message to its nearest node to serve as the processing center for future rounds.

Our assumption regarding PSO application not only helps in the swarm computation, but also makes the application of PSO Semi-Distributed (PSO-SD), unlike the research carried out so far in the related area. The implementation of the swarm intelligence method is described in the following section.

\section{Proposed cluster head selection technique using PSO}

We have modeled a novel usage of particle swarm optimization in cluster head selection, which makes the algorithm semi-distributed and comparatively more energyefficient. In order to accomplish the purpose, we have proposed a novel fitness function for PSO on the basis of distance, energy, node degree and head count of the sensor nodes in a cluster.

\section{Cluster-based particle swarm optimization}

It is assumed that $\mathrm{S}$ is the swarm space $\left(S \subset R^{2}\right)$ with $f: S \rightarrow G \subseteq R$ as the fitness or the cost function. The fitness function is used to select the optimum position for the cluster head. The point coordinates (separated by predefined position shift) within the swarm region are regarded as the population of points or particles. Let 
$\Sigma=\left\{x_{1}, x_{2}, \ldots, x_{N}\right\}$ be the set of all the particles considered for experimentation in the swarm $S$. If there are $N$ particles (candidate solutions) in the swarm, then each particle possesses a position vector $\left(x_{i}\right)$ and velocity vector $\left(v_{i}\right)$ given by:

$$
\begin{aligned}
& x_{i}=\left(x_{i 1}, x_{i 2}, \ldots, x_{i M}\right)^{T} \\
& v_{i}=\left(v_{i 1}, v_{i 2}, \ldots, v_{i M}\right)^{T}
\end{aligned}
$$

where, $i=1,2, \ldots, N$ and $M$ represents the dimension. Moreover, $x_{i j}(t)$ and $v_{i j}(t)$ signifies the $i$ th particle position and velocity in $j t h$ dimension during the time instant $t$. To track the global best positioning, $n_{C A}$ maintains the local best positions of the particles in the set $\Pi=\left\{p_{1}, p_{2}, \ldots, p_{N}\right\}$ which contains the best positions of all the particles ever visited.

$$
p_{i}=\left(p_{i 1}, p_{i 2}, \ldots, p_{i M}\right)^{T}
$$

Also, the local best position of ith particle and overall global best location (with respect to all particles in the swarm within a cluster) at time $t$ are denoted as $p_{i}(t)$ and $p_{g}(t)$ respectively.

$$
\begin{aligned}
& p_{i}(t)=\underset{t}{\arg \min } f_{i}(t) \\
& p_{g}(t)=\underset{i}{\arg \min } f\left(p_{i}(t)\right)
\end{aligned}
$$

The PSO algorithm is executed over a number of generations (or iterations) to explore maximum possible accuracy in finding the cluster head locations. Therefore, $n_{C A}$ uses the following equations to update the particle position and velocities:

$$
\begin{aligned}
v_{i j}(t+1) & =\omega(t) \nu_{i j}(t)+\left[c_{c o g} r_{1} p_{i j}(t)-x_{i j}(t)\right]+\left[c_{s o c} r_{2}\left(p_{g j}(t)-x_{i j}(t)\right)\right] \\
x_{i j}(t+1) & =x_{i j}(t)+v_{i j}(t+1)
\end{aligned}
$$

In the above equation, $t=0 \ldots T_{\max }$ and $T_{\max }$ represents the maximum number of generations. Also $r_{1}$ and $r_{2}$ are the random variables uniformly generated between [0,1]. The cognitive and social parameters, denoted by $c_{c o g}$ and $c_{s o c}$, defines the magnitude of search in the swarm. $\omega(t)$ is the inertia weight that is used for reducing the effect of previous particle velocity on the current generation. Absence of which may result in unusual drifts from the best achievable positions.

$$
\omega(t)=\omega_{\text {up }}-\left(\omega_{\text {up }}-\omega_{\text {low }}\right) \frac{t}{T_{\max }}
$$

The local best value is updated according to whether the new position is more fittable than the previous best position of that particle.

$$
p_{i}(t+1)= \begin{cases}x_{i}(t+1), & \text { if } f\left(x_{i}(t+1)\right) \leq f\left(p_{i}(t)\right) \\ p_{i}(t), & \text { otherwise }\end{cases}
$$

\section{Proposed fitness function for PSO}

Finally, in this section we define our proposed fitness function for effective execution of the cluster-based PSO. The main objective of the function is to optimize the combined effect of average distance from the sensors in a cluster, residual energy, node 
degree and head count (i.e. number of times a sensor node served as cluster head). The fitness function, represented as $f\left(x_{i}(t)\right)$ for the $i$ th particle is specified in the following equation:

$$
f\left(x_{i}(t)\right)=\text { optimize } \quad\left(\alpha_{1} X_{1}+\alpha_{2} X_{2}+\alpha_{3} X_{3}+\left(1-\alpha_{1}-\alpha_{2}-\alpha_{3}\right) X_{4}\right)
$$

Subject to:

$$
\begin{aligned}
& X_{1}=\sum_{\substack{\forall n_{j} \in C_{k} \\
x_{i} \in S}}\left\{\frac{\| n_{j}, x_{i}||}{\left|C_{k}\right|}\right\} \\
& X_{2}=\sum_{\substack{i=1 \\
x_{i} \in S}}^{N} E\left(p_{i}\right) / \sum_{\substack{j=1 \\
n_{j} \in C_{k}}}^{\left|C_{k}\right|} E\left(n_{j}\right), E_{\min } \leq E\left(n_{j}\right) \leq E_{\max } \\
& X_{3}=N_{\operatorname{deg}}\left(p_{i}\right), 0<\alpha_{1}, \alpha_{2}, \alpha_{3}<1 \\
& X_{4}=1 / H\left(p_{i}\right), H\left(p_{i}\right) \geq 1, \text { and } \alpha_{1} \leq \alpha_{2} \leq \alpha_{3}
\end{aligned}
$$

In the above equation $\alpha_{1}, \alpha_{2}, \alpha_{3}$ are the weightage parameters. In our optimization function we provide comparatively more valuation to the residual energy associated with the particle $p_{i}$. The sensor $\left(n_{j}\right)$ must have its energy level within the range $\left[E_{\min }, E_{\max }\right]$, or else the node is filtered out and hence not selected for comparison with other nodes in particle $p_{i}$. Moreover, $E\left(p_{i}\right), N_{\mathrm{deg}}\left(p_{i}\right)$ and $H\left(p_{i}\right)$ represents the energy, node degree and head count associated with the particle $p_{i}$. Also, $n_{j}$ is the $j t h$ node of $k$ th cluster $\left(C_{k}\right)$ and $\left|C_{k}\right|$ denotes the total number of nodes in the respective cluster. The Euclidean distance between node $n_{j}$ and particle $p_{i}$ is represented by notation $n_{j}, x_{i}$. It is clear from the equation that $\chi_{1}$ is the average distance of particle $p_{i}$ from all other sensors in the cluster and $\chi_{2}$ is the measure of particle energy w.r.t. other nodes. The $\chi_{3}$ parameter refers to the node degree associated with particle $p_{i}$. This criterion helps to select the node around the particle with highest degree. Moreover, the number of neighbors for a sensor can be easily determined with help of in-built commands of network simulator. The sensor that is connected to more number of nodes reflects greater efficiency in receiving more packets easily. $\chi_{4}$ is the probability of choosing a node in particle $p_{i}$ on the basis of its head count. The head count is basically the frequency of a node of becoming cluster head so far. As the head count increases, the probability of its selection as cluster head decreases by certain magnitude.

Finally, at the end of each round (i.e. on completion of $T_{\max }$ number of generations), the particle whose attributes optimizes the objective function is chosen as the global best position for the head node. The sensor nearest to the global best location is elected as the $\mathrm{CH}$ for the current round. The selected node acts as $\mathrm{CH}$ until its energy drops beyond a specified level. After which the current $\mathrm{CH}$ informs $n_{C A}$ to initiate the PSO computation for the next round of cluster head selection.

\section{Packet retransmissions computation}

Our energy consumption model considers the energy used in successful transmissions, receptions as well as the number of times a packet is collided (retransmissions). For 
this purpose, we have computed the average number of times a packet is retransmitted due to collision before successfully receiving at the destination (i.e. cluster head).

We assume that in average there are $n$ links between member nodes and cluster head. Therefore in ideal case, exactly $n$ transmissions would be required to successfully deliver one packet to $\mathrm{CH}$. However, owing to the inherent nature of wireless communication in sensor network, packet losses are quite inevitable. The communication path remains highly vulnerable to network disturbances due to several factors. This increases the likelihood of retransmissions of the collided packets. In order to assess the energy consumption, we have theoretically computed the packet retransmissions within the cluster. Hence, the significant contributions included in this section are summarized as follows:

- Estimation of the average intra-cluster distance

- Determination of the number of communication links between the member nodes and the cluster head

- Derivation of average retransmissions of the collided packets

- Justification of the theoretical computation with simulated performance

The PSO-based cluster aims at selecting cluster heads that reduces the intra-cluster distance and minimizes the redundant packet transmissions. As a result of which large amount of energy wasted in communication can be preserved. In best case, CHs are ensured to be located within the COM area. However, due to the depletion of sensor energy, the position of selected CHs might not be always within the suitable region, yet assuming ideal behavior we can approximate the actual performance. In the subsequent research, we presumed ideal conditions and obtained an estimation of the distance.

\section{Distance estimation of sensors to cluster head}

In this segment we derive the expectation of the distance $d$ from the cluster member to its cluster head within a particular cluster region. In the following equation, the cluster head is assumed to be in the middle (i.e. center of mass) of the cluster $\left(x_{c}=0, y_{c}=0\right)$.

$$
\begin{aligned}
E[d] & =\iint \sqrt{\left(\left(x-x_{c}\right)^{2}+\left(y-y_{c}\right)^{2}\right)} \rho(x, y) d x d y \\
& =\iint \sqrt{\left(x^{2}+y^{2}\right)} \rho(x, y) d x d y
\end{aligned}
$$

Now, on converting equation (12) to polar form and putting $\sqrt{x^{2}+y^{2}}=r$, we get:

$$
E[d]=\iint r \rho(r, \theta) r d r d \theta=\rho \iint r^{2} d r d \theta
$$

The approximated cluster radius found in equation (1) is used as a limit to integrate the distance estimation as following:

$$
E[d]=\rho \int_{0}^{2 \pi} \int_{0}^{R_{s} / \sqrt{k}} r^{2} d r d \theta=\rho \int_{0}^{2 \pi}\left[\frac{r^{3}}{3}\right]_{0}^{R_{s} / \sqrt{k}} d \theta=\frac{2 \pi \rho R_{s}^{3}}{3 k \sqrt{k}}
$$


Further, we abide by the assumption that the density of the sensors within cluster is uniform, which implies:

$$
\rho=\frac{1}{\pi R_{s}^{2} / k}=\frac{k}{\pi R_{s}^{2}}
$$

On providing the value of $p$ in equation (14), we have:

$$
E[d]=\frac{2 \pi R_{s}^{3}}{3 k \sqrt{k}} \times \frac{k}{\pi R_{s}^{2}}=\frac{2 R_{s}}{3 \sqrt{k}}
$$

Finally, the estimated number of hops required to be traversed by a node in order to send data to the cluster head, can be computed as following with $r_{\min }$ as the minimum permissible transmission range of a sensor node:

$$
h=\frac{E[d]}{r_{\min }}=\frac{2 R_{s}}{3 r_{\min } \sqrt{k}}
$$

\section{Expected number of retransmission attempts}

We know that clustered sensor members having their communication ranges overlapped are regarded as neighbors. This implies that communication path exists between the neighboring nodes, which further results in existence of an aggregation tree in every cluster with the cluster head $(\mathrm{CH})$ as the root. The $\mathrm{CH}$ is also regarded as the aggregation center, which aggregates the data gathered from the member nodes for a given period of time. The transmission of data to the $\mathrm{CH}$ follows the path in the aggregation tree. We assume that there are $h$ hops or links between the source node and $\mathrm{CH}$. Also, every link between sensors possesses link failure probability $\left(p_{l k}\right)$, which refers to the susceptibility of the channel to failures, due to different reasons.

Figure 4 shows the transmission process of a packet from the sensor $s\left(o^{\prime}, r_{\min }\right)$ to the cluster head. It is clear from the figure that for a tree of $h$ links, the number of transmissions required for one successful sending of packet to $\mathrm{CH}$ is also $h$. Now, the probability of $h$ successful transmission for one successful end-to-end data delivery towards $\mathrm{CH}$ is $\left(1-p_{l k}\right)^{h}$. Also, the probability of at least one failed transmission, leading to unsuccessful data delivery, is $1-\left(1-p_{l k}\right)^{h}$. Let us consider a random variable $Y$ which denotes the number of successful data delivery attempts. Further, $(\eta-1)$ failures followed by one successful attempt, satisfies geometric distribution. This can be written as follows:

$$
P[Y=\eta]=\left[1-\left(1-p_{l k}\right)^{h}\right]^{\eta-1} \times\left(1-p_{l k}\right)^{h}
$$

We further derive the estimated number of transmissions required for worst case network performance. This criterion helps to monitor the effectiveness of our proposed approach even if packet retransmissions are involved. Therefore, the expected number of attempts leading to one successful delivery of packet can be computed as following: 


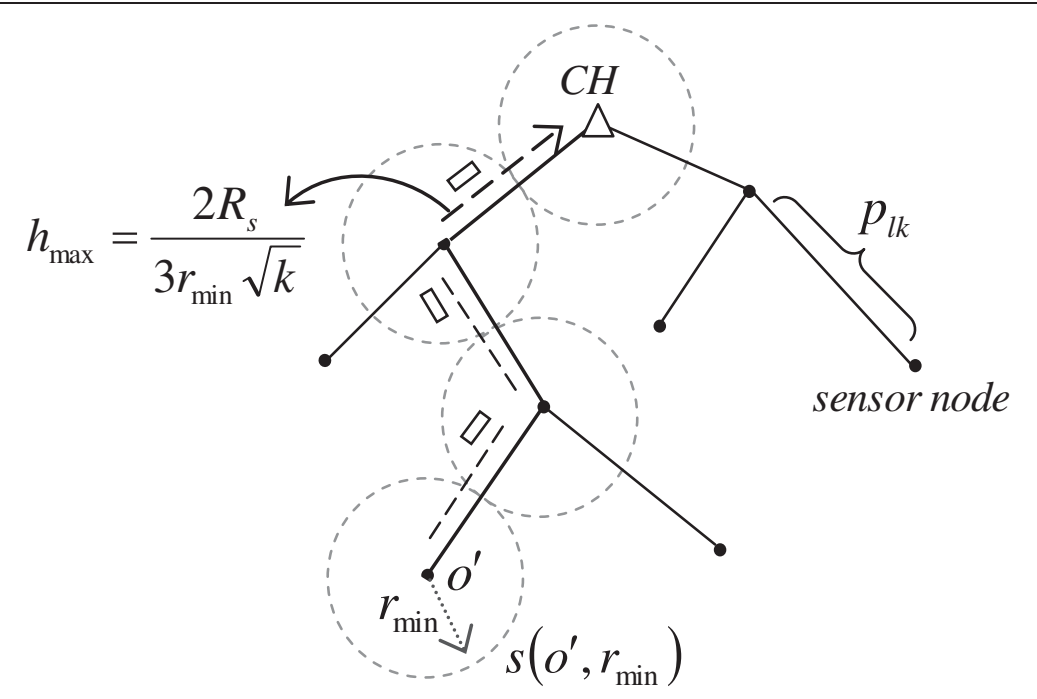

Figure 4 Packet Transmission to Cluster Head.

$$
\begin{aligned}
& E[\eta]=\sum_{\eta=1}^{\infty} \eta \times P[Y=\eta]=\sum_{\eta=1}^{\infty} \eta \times\left[1-\left(1-p_{l k}\right)^{h}\right]^{\eta-1} \times\left(1-p_{l k}\right)^{h} \\
& =\frac{\left(1-p_{l k}\right)^{h}}{1-\left(1-p_{l k}\right)^{h}} \times \sum_{\eta=1}^{\infty} \eta \times\left[1-\left(1-p_{l k}\right)^{h}\right]^{\eta}=\frac{\left(1-p_{l k}\right)^{h}}{1-\left(1-p_{l k}\right)^{h}} \times \frac{1-\left(1-p_{l k}\right)^{h}}{\left[1-\left(1-\left(1-p_{l k}\right)^{h}\right)\right]^{2}} \\
& \Rightarrow E[\eta]=\frac{1}{\left(1-p_{l k}\right)^{h}}
\end{aligned}
$$

However, the estimated number of hops between sensor nodes and its $\mathrm{CH}$ is specified in equation (17). So, replacing the value of $h$ we finally get:

$$
E[\eta]=\frac{1}{\left(1-p_{l k}\right)^{2 R_{s} / 3 r_{\min } \sqrt{k}}}
$$

Equation (21) provides an average perception of needed retransmissions which is not computed by the individual sensors. This estimation is only derived to assist the determination of the average energy consumption in the network, as described in the next section. Moreover, the behavioral analysis of our theoretical model with respect to actual simulation results is provided in the following.

The graph in Figure 5 clearly depicts that as the average number of nodes increase in a cluster, the trend of packet retransmissions also increases. The escalation is because of the fact that, with the rise in number of nodes the cluster becomes denser and therefore the number of links towards cluster head increases. This introduces more chances of packet losses due to collisions. The result highlights that our theoretical computation of probable number of retransmissions in a cluster, fairly provides a good estimate of the simulated behavior. Furthermore, the graphical outcome justifies the appropriateness of our mathematical derivation of packet retransmissions. 


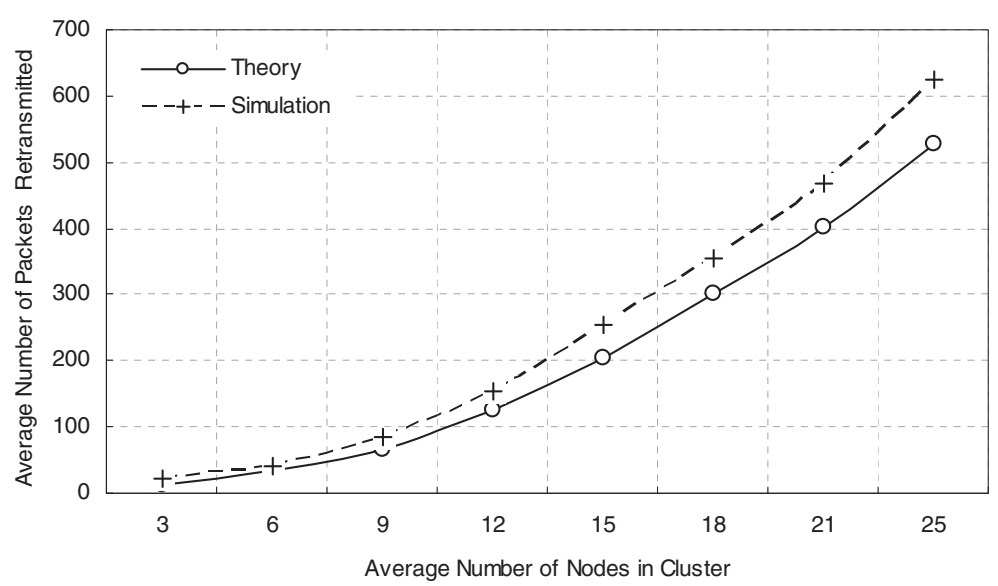

Figure 5 Average Packet Retransmissions in Cluster.

\section{Proposed energy consumption model}

Sensor nodes mainly dissipate radio energy in form of electronics and amplifier energy. The energy consumed in the radio electronics $\left(E_{\text {elec }}\right)$ fundamentally depends on how efficiently the signal is encoded, modulated and filtered. The energy dissipation rate in the radio amplifier $\left(\epsilon_{\text {amp }}\right)$ is directly proportional to $d^{\gamma}$. Here, $d$ is regarded as the distance between the source and destination node, and $\gamma$ is the path loss component. The path loss experienced by signal can be different depending upon the power loss model employed.

There are two types of power loss encountered by a signal: free-space $\left(\epsilon_{f s} ; \gamma=2\right)$ and multi-path fading $\left(\epsilon_{m p} ; \gamma=4\right)$. For our simulation the sensors are distributed in the network in such a way that the small-sized clusters are formed. Therefore, the energy dissipation is asumed to follow the Friss free-space model, i.e. the power loss in the radio electronics and amplifier is proportional to the square of the distance $\left(d^{2}\right)$ between the member node (source) and cluster head (destination). Moreover, the expected value of $d^{2}$, represented by $E\left[d^{2}\right]$ is obtained as following:

$$
\begin{aligned}
E\left[d^{2}\right] & =\iint\left(x^{2}+y^{2}\right) \rho(x, y) d x d y \\
& =\rho \int_{0}^{2 \pi} \int_{0}^{R_{s} / \sqrt{k}} r^{3} d r d \theta=\frac{\pi \rho R_{s}^{4}}{2 k^{2}}=\frac{\pi \rho R_{s}^{4}}{2 k^{2}}
\end{aligned}
$$

In order to transmit $m$-bit length of packet over a distance $d$, the energy used by a sensor node is given by:

$$
e_{t x}(m, d)=\left\{\begin{array}{lr}
m \lambda E_{\text {elec }}+m \epsilon_{f_{s}} d^{2} & d<v_{o} \\
m \lambda E_{\text {elec }}+m \epsilon_{m p} d^{4} & d \geq v_{o}
\end{array}\right.
$$

where $v_{o}$ is the threshold distance, beyond which the strength of the signal is affected by multi-path fading. Moreover, the energy required by a sensor to receive one $m$-bit packet is:

$$
e_{r x}(m)=m E_{\text {elec }}
$$


Finally, the energy used $\left(\xi_{\text {node }}\right)$ by a sensor node to perform transmission-reception operations accompanied with occasional sleep phases, can be determined by the following equation:

$$
\begin{aligned}
\xi_{\text {node }} & =\left(1-\beta_{s}\right)\left[e_{t x}(m, d)+e_{r x}(m)\right]+\beta_{s} e_{s p} \\
& =\left(1-\beta_{s}\right)\left[m \lambda E_{\text {elec }}+\left(m \epsilon_{f s} \times \frac{R_{s}^{2}}{2 k}\right)+m E_{\text {elec }}\right]+\beta_{s} e_{s p}
\end{aligned}
$$

Here, $\beta_{s}$ is the sleep probability of a sensor node calculated on the basis of the outcome of randomized scheduler. Besides the power expended by an individual sensor, the total energy required for successful delivery of a packet fundamentally relies on the estimated number of hops to be traversed to reach the destination and the expectation of the packet retransmissions required. So, the total energy in delivering a packet $\left(\xi_{p k t}\right)$ can be expressed as following:

$$
\xi_{p k t}=h \times \xi_{\text {node }} \times E[\eta]
$$

On replacing the values of $h, E[\eta]$ and $\xi_{\text {node }}$ from equations (17, 21 and 25), we get the following result for energy expended in a packet delivery operation:

$$
\begin{aligned}
\xi_{p k t} & =\frac{2 R_{s}}{3 r_{\min } \sqrt{k}} \times\left\{\left(1-\beta_{s}\right)\left[m \lambda E_{\text {elec }}+\left(m \epsilon_{f s} \times \frac{R_{s}^{2}}{2 k}\right)+m E_{\text {elec }}\right]+\beta_{s} e_{s p}\right\} \\
& +\frac{1}{\left(1-p_{l k}\right)^{2 R_{s} / 3 r_{\min } \sqrt{k}}}
\end{aligned}
$$

Further, the overall energy consumption $\left(\xi_{\text {total }}^{(\text {overall })}\right)$ made by all the sensors within a cluster having Poisson distributed sensors and expected node density as $\rho$, is expressed as:

$$
\begin{aligned}
\xi_{\text {total }}^{(\text {overall })} & =\sum_{n=0}^{\infty} n \xi_{\text {node }} \times \frac{\left(\rho \pi R^{2}\right)^{n}}{n !} \times e^{-\rho \pi R^{2}} \\
& =\xi_{\text {node }} \sum_{n=0}^{\infty} n \times \frac{\left(\rho \pi R^{2}\right)^{n}}{n !} \times e^{-\rho \pi R^{2}}=\xi_{\text {node }} \times \rho \pi R^{2}
\end{aligned}
$$

Therefore, equation (28) clearly explains that the overall energy consumption depends upon the expected number of retransmissions, average number of hops or links, active probability of a sensor and the expected number of nodes in the cluster, i.e. $\rho \pi R^{2}$.

\section{Simulation \& performance evaluation}

The network scenario is designed and implemented using Network Simulator (NS2.34) $[22,23]$. The simulator executes the PSO method for cluster head selection and tracks the rate of energy consumption of the sensors. In our 200-node sensor network, there are specifically three types of sensors (excluding the base station) - sensing nodes, assistant nodes and the cluster head nodes. All the nodes are considered homogeneous but are assigned different tasks to perform. Such distribution not only coordinates and balances the operational load within the cluster, but also results in improved management and extended network lifetime. The sensing nodes are deployed with the task of sensing the monitored entity and uploading the samples to the cluster head (when demanded). The cluster head schedules data collection periods and performs 
aggregation of the gathered data before transmitting them to the sink. In addition, the assistant node is employed for applying PSO to cluster head selection. In this section, we will evaluate our simulation results in terms of several parameters, for instance network lifetime (number of active nodes), energy consumption and average number of packets delivered. The network simulation parameters and swarm optimization parameters are defined in Table 1.

In Figures 5 and 6 the convergence rate of our proposed PSO-Semi Distributed (PSO-SD) fitness function is provided for the theoretical model and simulation as well. It is evident from the graph that till 100 generations, the actual implementation (i.e. simulation) deviates from the theory by some magnitude. It is because of the fact that, the PSO operations initiate with the collection of certain details (location, energy, node degree, head count) from the sensor nodes in a cluster. During the simulation, information collection phase experiences various impediments, such as - collisions and transmission delays, which actually results in deviation.

The sensor network lifetime is evaluated in Figure 7 in terms of number of nodes that manage to remain alive as the network lifetime advances. Clearly, our proposed work, PSO-SD achieves comparatively better extension to network lifetime. The reason behind the significant achievement is the optimized localization of the cluster heads by swarm optimization. The PSO-C (PSO-clustering) protocol deteriorates because of the fact that PSO operations are executed in an entirely centralized way at the base station. The transmission of the sensor information to the sink at the beginning of every $\mathrm{CH}$ selection round introduces unwanted delays and energy waste. Moreover, LEACH-C suffers largely because of poor network clustering and cluster head selection. The

Table 1 Network simulation parameters

\begin{tabular}{ll}
\hline PARAMETER & VALUE \\
\hline Sensor network dimension & $1000 \times 1000$ sq. meters \\
Base station location & At $(600,600)$ \\
Sensor radius $(r)$ & 115 meters \\
Transmission power $\left(e_{t x}\right)$ & 12.910 watts \\
Reception power $\left(e_{r x}\right)$ & 11.081 watts \\
Sleep power $\left(e_{s p}\right)$ & 0.00214 watts \\
Packet Length $(m)$ & 14 bytes \\
Weightage parameters $\left(a_{1}, a_{2}, a_{3}\right)$ & $(0.3,0.4,0.2)$ \\
Energy required in radio electronics $\left(E_{\text {elec }}\right)$ & $50 \mathrm{~nJ} / \mathrm{bit}$ \\
Energy required in radio electronics $\left(\epsilon_{\text {amp }}\right)$ & $10 \mathrm{pJ} / \mathrm{bit} / \mathrm{m}^{2}$ \\
Initial sensor energy & 150 joules \\
Number of generations $\left(T_{\text {max }}\right)$ & 250 \\
Number of particles $(N)$ & 20 \\
Cognitive parameter $\left(c_{\text {cog }}\right)$ & 2 \\
Social parameter $\left(c_{\text {soc }}\right)$ & 2 \\
Inertia weight $(\omega)$ & from 1.2 to 0.4 \\
Routing protocol & Destination Sequence Distance- \\
Total sensor nodes deployed & Vector (DSDV protocol \\
Total simulation period & 200 \\
\hline & 800 seconds \\
\hline
\end{tabular}




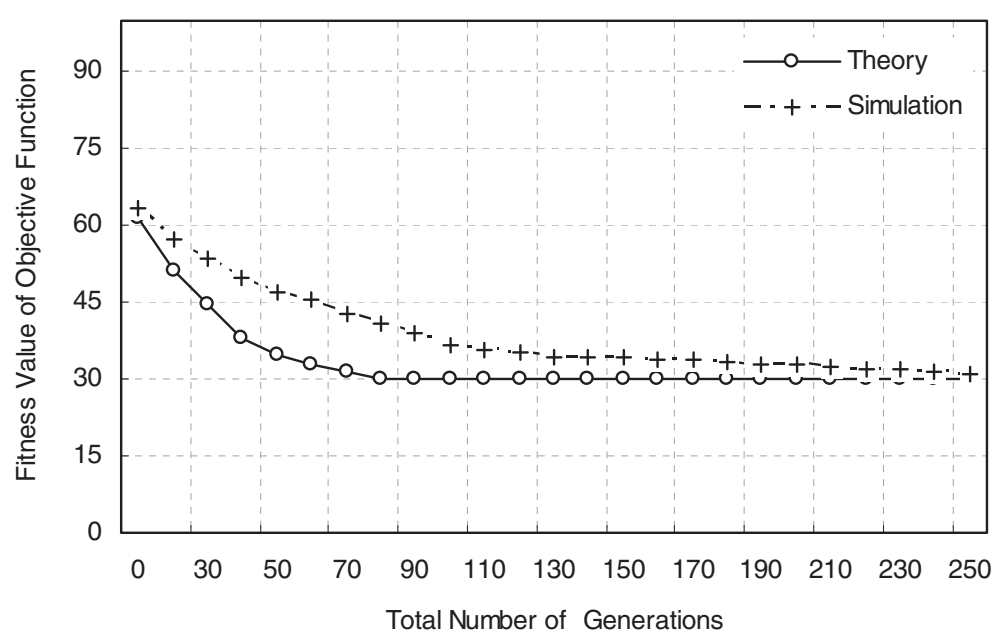

Figure 6 Convergence Rate of the PSO Objective Function.

member nodes of a cluster need to overpass long distances in order to reach the $\mathrm{CH}$, thereby leading to energy exhaustion.

The average communication supported by the sensors within the network lifetime is highlighted in Figure 8. PSO-SD shows considerable increase in the average number of packets delivered. The trend increases till $t=425 \mathrm{sec}$, after which it gradually drops due to energy insufficiency in the network. However, the rate of successful packet delivery still retains greater performance than the referenced counterparts. LEACH-C suffers the most as poorly managed clusters fail to communicate the packets to the head node. Also, it does not take residual energy into account while selecting the cluster head. Though, PSO-C survives to support packets transmission but it ultimately perishes due to the centralized execution of swarm optimization.

Further, in Figure 9 we study the total number of cluster head selection rounds supported by the active nodes in the network. This graph assists in evaluating the overall cost of applying PSO to the cluster head selection procedure. From the large number of selection rounds (over 1200 rounds) supported by our proposed technique, it can be

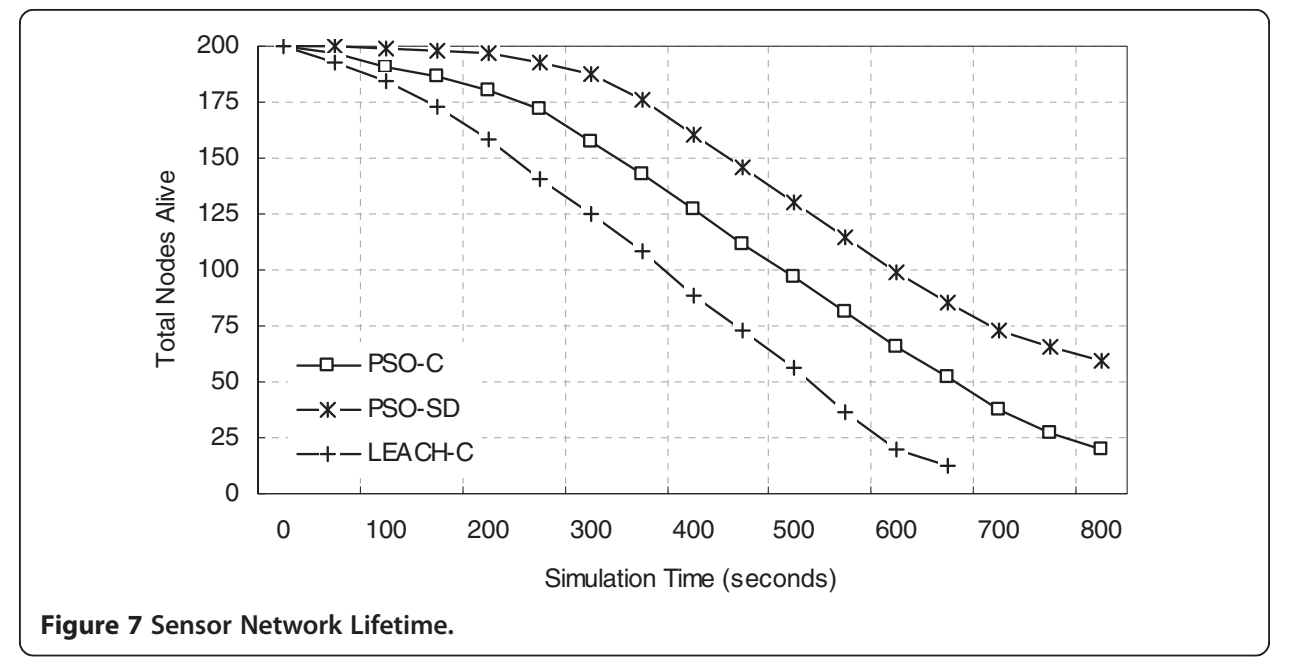




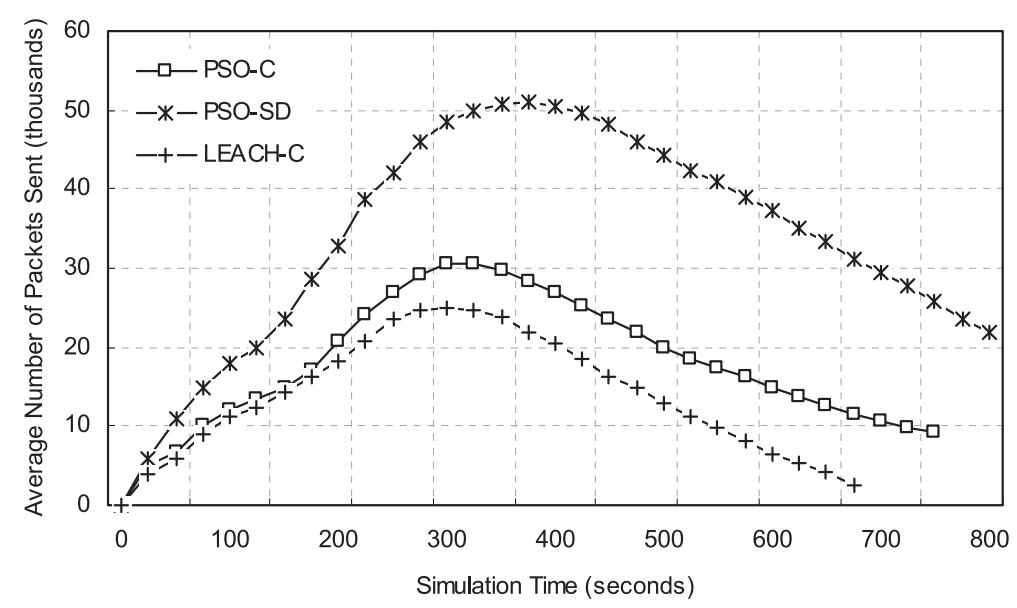

Figure 8 Average Number of Packets Transmission over Simulated Time Frame.

easily deduced that cost of the PSO operations completely outweighs the extended lifetime achieved. On the contrary, $\mathrm{LEACH}-\mathrm{C}$ degrades at a faster rate owing to its inefficient methodologies for network partitioning and head node selection. Moreover, PSO$\mathrm{C}$ also shows gradual decrement in the plot. Such observation is expected because the member nodes remain busy most of the times in forwarding sensor informations to sink rather than monitoring the environment.

Figure 10 illustrates the amount of energy consumed by the sensors over a simulation period of 800 seconds. The graph clearly establishes the effectiveness of our proposed PSO-SD in delivering maximum sensor operations in an energy-efficient way. The rate at which the residual energy of the sensors is replenished with PSO-SD is apparently lower than the PSO-C and LEACH-C protocols respectively. The reason behind is that our proposed PSO-based cluster scheme is semi-distributed and well-managed. However, the other protocols either lack proper cluster head control (LEACH-C) or decentralization concepts (PSO-C), due to which the battery-based sensors run out of power rapidly.

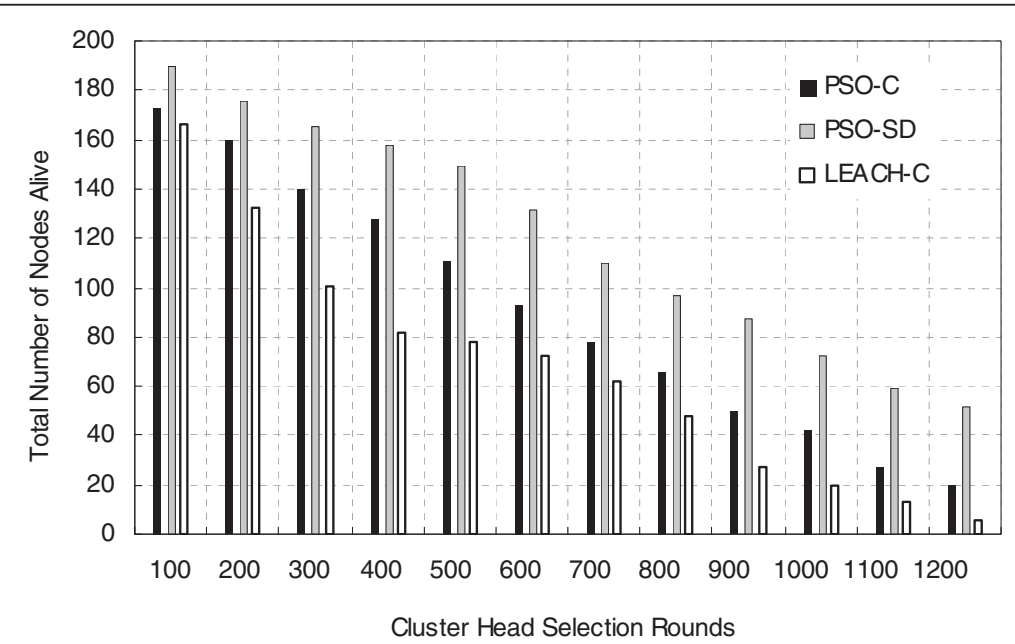

Figure 9 Number of PSO-based Cluster Head Selection Rounds Supported by the Sensor Network. 


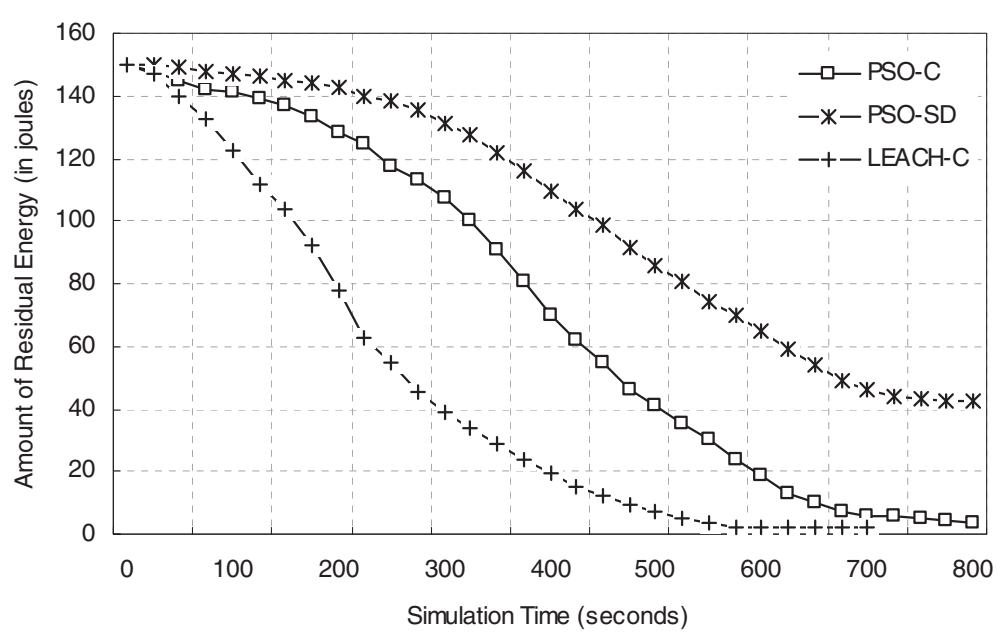

Figure 10 Average Energy Consumption in the Network.

\section{Conclusion \& future work directions}

The energy constraints in sensors pose serious challenges to the developers of sensor network. In order to improve the network performance several energy-aware clusterbased techniques are designed. The issue of optimal cluster head selection is often formulated as an optimization problem. Particle Swarm Optimization (PSO) is such a method which is known for its easy implementation and fast convergence.

In this paper, we have applied PSO for selecting the cluster heads by optimizing certain performance criteria. Our cluster-based strategy enhances network performance as compared to certain existing techniques. The proposed PSO-SD reduces the intracluster distance from the cluster members to the cluster head. The location of the cluster head is optimized by the proposed objective function used by PSO. Moreover, retransmissions computation for collided packets also assists in the derivation of overall energy consumption in the network. The simulation results illustrate that our PSO-SD delivers better performance than its comparatives in terms of lifetime, energy consumption and average number of packets communicated to the base station. There are several promising research directions that can be projected from our present contribution. Our future work includes the implementation of sensor mobility in higher dimensional region of interest. Moreover we would also focus on the distributed PSO-application in heterogeneous sensor networks.

\section{Competing interests}

The authors declare that we have no significant competing financial, professional or personal interests that might have influenced the performance or presentation of the work described in this manuscript.

\section{Authors' contribution}

The authors have applied Particle Swarm Optimization for locating optimal position of cluster heads within the cluster on the basis of proposed objective function. The contribution ultimately reduced the intra-cluster communication distance thereby minimizing the overall energy consumed in packet transmissions. Furthermore, the proposed strategy is implemented within the cluster rather than base station, which makes it a semi-distributed and energy saving approach. All authors have read and approved the final manuscript. 
Received: 30 November 2011 Accepted: 8 May 2012

Published: 16 July 2012

\section{References}

1. Akyildiz IF, Su W, Sankarasubramaniam Y, Cayirci E (2002) Wireless sensor networks: a survey. J of Compute Networks 38(4):393-422..

2. Yong-Min L, Shu-Ci W, Xiao-Hong N (2009) The architecture and characteristics of wireless sensor networks, IEEE International Conference on Computer Technology and Development. Kota Kinabalu, Malaysia, pp 561-565, 13-15 November 2009.

3. Zeng Y, Sreenan CJ, Xiong N, Yang LT, Park JH (2010) Connectivity and coverage maintenance in wireless sensor networks. J Supercom Springer 52(1):23-46.

4. Parsopoulos KE, Vrahatis MN (2010) Particle swarm optimization and intelligence: advances and applications. Information Science Reference, IGI Global, pp 1-41.

5. Panigrahi BK, Shi Y, Lim M-H (2011) Handbook of swarm intelligence: concepts, principles and applications. Springer, Berlin, Germany, pp 3-292.

6. Kulkarni RV, Venayagamoorthy GK (2011) Particle swarm optimization in wireless-sensor networks: a brief survey. IEEE Transactions on Systems, Man, and Cybernatics-Part C. Appl Rev 41(2):262-267.

7. Djeffal F, Lakhdar N, Meguellati M, Benhaya A (2009) Particle swarm optimization versus genetic algorithms to study the electron mobility in wurtzite GaN-based devices. Solid-State Electr J Elsevier 53(9):988-992.

8. Katari V, Malireddi S, Bendapudi SKS, Panda G (2008) Adaptive Nonlinear System Identification using Comprehensive Learning PSO. IEEE 3rd international symposium on communications, control, and signal processing, Malta, Europe, pp 434-439, 12-14 March 2008.

9. Latiff NMA, Tsemenidis CC, Sheriff BS (2007) Energy-aware clustering for wireless sensor networks using particle swarm optimization, The 18th Annual IEEE International Symposium on Personal. Indoor and Mobile Radio Communications, Athens, Greece, pp 1-5, 3-7 September 2007.

10. Heinzelman W, Chandrakasan A, Balakrishnan H (2000) Energy-efficient communication protocols for wireless microsensor networks. IEEE Proceedings of the 33rd Hawaii International Conference on System Sciences, Island of Maui, Hawaii, pp 1-10, 4-7 January 2000.

11. Heinzelman W, Chandrakasan A, Balakrishnan H (2002) An application-specific protocol architecture for wireless microsensor networks. IEEE Trans Wirel Commun 1(4):660-670.

12. Yang E, Erdogan AT, Arslan T, Barton N (2007) An improved particle swarm optimization algorithm for powerefficient wireless sensor networks. ECSIS Symposium on Bio-inspired, Learning, and Intelligent Systems for Security, Edinburgh, UK, pp 76-82, 9-10 August 2007.

13. Cao X, Zhang H, Shi J, Cui G (2008) Cluster heads election analysis for multi-hop wireless sensor networks based on weighted graph and particle swarm optimization. Fourth International Conference on Natural Computation, Jinan, China, pp 599-603, 14 March 2008.

14. Low KS, Nguyen HA, Guo H (2008) A particle swarm optimization approach for the localization of a wireless sensor network. IEEE International Symposium Industrial Electronics (ISIE'08), Cambridge, UK, pp 1820-1825, 29 June - 2 July 2008.

15. Pradhan PM, Baghel V, Panda G, Bernard M (2009) Energy efficient layout for a wireless sensor network using multi-objective particle swarm optimization. IEEE International Advance Computing Conference, Patiala, India, pp 65-70, 6-7 March 2009.

16. Aziz NABA, Mohemmed AW, Alias MY (2009) A wireless sensor network coverage optimization algorithm based on particle swarm optimization and voronoi diagram, IEEE International Conference on Networking. Sensing and Control, Okayama City, Japan, pp 602-607, 26-29 March 2009.

17. Deosarkar BP, Yadav NS, Yadav RP (2009) A particle swarm approach for uniform cluster distribution in data centric wireless sensor networks. World Congress on Nature \& Biologically Inspired Computing, Coimbatore, India, pp 766-771, 9-11 December 2009.

18. Ismail WZW, Manaf SA (2010) Study on coverage in wireless sensor network using grid based strategy and particle swarm optimization. IEEE Asia Pacific Conference on Circuits and Systems, Kuala Lumpur, Malaysia, pp 1175-1178, 6-9 December 2010.

19. Hu B, Liu S, Li H (2009) Mobile beacon node path scheme based on particle swarm optimization in wireless sensor networks. Ninth International Conference on Electronic Measurement and Instruments, Beijing, China, pp 3-306-3-309, 16-19 August 2009.

20. Singh B, Lobiyal DK (2012) Energy-aware cluster head selection using particle swarm optimization and analysis of packet retransmissions in WSN, 2nd International Conference on Computer, Communication. Control and Information Technology, Kolkata, India, pp 1-6, 25-26 February.

21. Xiaoling W, Lei S, Jin W, Cho J, Lee S (2006) Energy-efficient deployment of mobile sensor network by PSO, The Eighth Asia Pacific Web Conference'06, Harbin, China,16-18 January 2006. Lecturer Notes in Computer Science (LNCS), Springer, pp 373-382.

22. Fall K, Varadhan K (2009) The NS manual. The VINT Project, Berkley, USA.

23. Issariyakul T, Hossain E (2009) Introduction to Network Simulator NS2. Springer, New York, USA.

doi:10.1186/2192-1962-2-13

Cite this article as: Singh and Lobiyal: A novel energy-aware cluster head selection based on particle swarm optimization for wireless sensor networks. Human-centric Computing and Information Sciences 2012 2:13. 